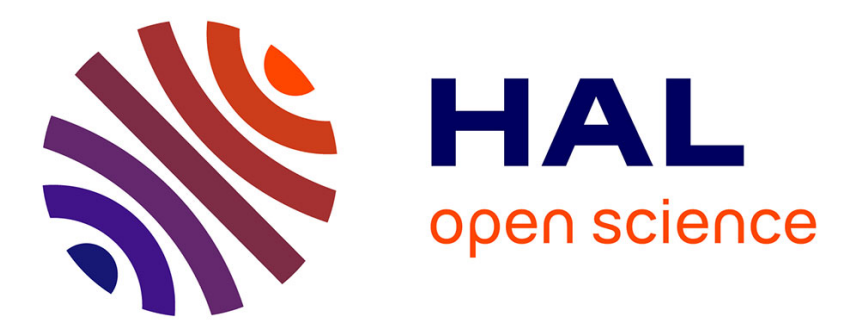

\title{
A formal quantifier elimination for algebraically closed fields
}

\author{
Cyril Cohen, Assia Mahboubi
}

\section{To cite this version:}

Cyril Cohen, Assia Mahboubi. A formal quantifier elimination for algebraically closed fields. Symposium on the Integration of Symbolic Computation and Mechanised Reasoning, Calculemus, Jul 2010, Paris, France. pp.189-203, 10.1007/978-3-642-14128-7_17 . inria-00464237v3

\section{HAL Id: inria-00464237 https://hal.inria.fr/inria-00464237v3}

Submitted on 29 Mar 2010

HAL is a multi-disciplinary open access archive for the deposit and dissemination of scientific research documents, whether they are published or not. The documents may come from teaching and research institutions in France or abroad, or from public or private research centers.
L'archive ouverte pluridisciplinaire HAL, est destinée au dépôt et à la diffusion de documents scientifiques de niveau recherche, publiés ou non, émanant des établissements d'enseignement et de recherche français ou étrangers, des laboratoires publics ou privés. 


\title{
A formal quantifier elimination for algebraically closed fields
}

\author{
Cyril Cohen, Assia Mahboubi \\ INRIA Saclay - Île-de-France \\ LIX École Polytechnique \\ INRIA Microsoft Research Joint Centre \\ [Cyril.Cohen|Assia.Mahboubi] @inria.fr
}

\begin{abstract}
We prove formally that the first order theory of algebraically closed fields enjoy quantifier elimination, and hence is decidable. This proof is organized in two modular parts. We first reify the first order theory of rings and prove that quantifier elimination leads to decidability. Then we implement an algorithm which constructs a quantifier free formula from any first order formula in the theory of ring. If the underlying ring is in fact an algebraically closed field, we prove that the two formulas have the same semantic. The algorithm producing the quantifier free formula is programmed in continuation passing style, which leads to both a concise program and an elegant proof of semantic correctness.
\end{abstract}

\section{Introduction}

Quantifier elimination is a standard way of proving the decidability of first order theories. In this paper, we investigate the formalization of quantifier elimination, and decidability for the first order theory of algebraically closed fields, inside the CoQ proof assistant [4]. The work does not address the problem of implementing a fast proof producing decision procedure. Our motivation is to enrich an existing hierarchy of algebraic structures $[8]$ with a decidability result. Beside automation, decidability validates case analysis on first-order statements, even in the context of a constructive development. In this work, we follow the proof given in standard references [2]. Yet we diverge from the usual expositions of the algorithm using continuation passing style to rephrase and prove quantifier elimination in a more elegant way.

The CoQ files for this formalization are available on line at the following URL : http://perso.crans.org/cohen/closedfields ${ }^{1}$.

The article is composed of three parts. First, we reduce quantifier elimination in discrete structures to the elimination of a single existential quantifier. We also build the boolean decision procedure resulting from quantifier elimination. Then, we establish an algebraic characterization of any existential formula with a single quantifier. Finally, we show how to compute a quantifier free formula from this characterization, using a continuation passing style formula transformation.

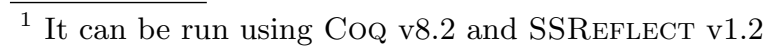




\section{Quantifier elimination and decidability}

\subsection{Preliminaries}

In this section we recall some standard definition, essentially following [11], and introduce some notations needed in the sequel.

Syntax : Signature, Terms, Formulas. In all what follows, we consider signatures of the form: $\Sigma=\mathcal{C} \cup \mathcal{F} \cup \mathcal{R}$, formed of a finite set $\mathcal{C}$ of constant symbols, a finite set $\mathcal{F}$ of function symbols with arity, and a finite set $\mathcal{R}$ of relation symbols with arity. Given such a signature $\Sigma$ and a countable set of variables $\mathcal{V}$, terms are inductively defined as: variables in $\mathcal{V}$ and constants in $\mathcal{C}$ are terms, other terms being of the form $f\left(t_{1}, \ldots, t_{n}\right)$ where $f \in \mathcal{F}$ is function with arity $n$ and $t_{1} \ldots t_{n}$ are terms. A term is closed if no variable occur in it. We write $\mathcal{T}(\Sigma, \mathcal{V})$ for terms, and $\mathcal{T}(\Sigma)$ for closed terms.

The atomic formulas of a signature $\Sigma$ are of the form $t_{1}=t_{2}$ where $t_{1}, t_{2}$ are any terms, and $R\left(t_{1}, \ldots, t_{n}\right)$ where $R \in \mathcal{R}$ is a relation with arity $n$. The first order language of $\Sigma$ is the set of all first order formulas with these atoms. First order formulas of $\Sigma$ are recursively defined by: atomic formulas are first order formulas, other formulas being of the form $\neg f, f_{1} \wedge f_{2}, f_{1} \vee f_{2},(\exists x, f),(\forall x, f)$, $f_{1} \Rightarrow f_{2}$, where $f, f_{1}, f_{2} \in F$ A formula is closed if no variable occurs in it. We write $\mathcal{F}(\Sigma, \mathcal{V})$ for formulas, and $\mathcal{F}(\Sigma)$ for closed formulas. We call theory over $\Sigma$ any set of closed formulas. We use the $\vdash$ predicate to denote provability: $T \vdash \psi$ means that $\psi$ is a first order consequence from formulas in $T$.

A theory $T$ admits quantifier elimination if, for every $\phi(x) \in \mathcal{F}(\Sigma, \mathcal{V})$, there exists $\psi(\boldsymbol{x}) \in \mathcal{F}(\Sigma, \mathcal{V})$ such that $\psi$ is quantifier free and

$$
T \vdash \forall \boldsymbol{x},((\phi(\boldsymbol{x}) \Rightarrow \psi(\boldsymbol{x})) \wedge(\psi(\boldsymbol{x}) \Rightarrow \phi(\boldsymbol{x})))
$$

However, we'll use a semantic characterization of quantifier elimination, defined in the end of then section about semantics.

Semantics : $\boldsymbol{\Sigma}$-structures, Models. For any signature $\Sigma=\mathcal{C} \cup \mathcal{F} \cup \mathcal{R}$, a $\Sigma$ structure is the pair of a set $E$ called the domain, and an interpretation function $I$ assigning an element of $E$ to each constant symbol in $\mathcal{C}$, a function $E^{n} \rightarrow E$ to each function symbol in $\mathcal{F}$ with arity $n$, and an $n$-ary relation on $E$ (i.e. a subset of $E^{n}$ ) to each relation symbol in $\mathcal{R}$ with arity $n$.

For any $\Sigma$-structure $A$, any term $t(\boldsymbol{x})$, and any list $e$ of values in the domain of $A$ at least as long as $\boldsymbol{x}$, we define inductively $[\boldsymbol{t}(\boldsymbol{x})]_{A, e}$ as

- if $\boldsymbol{t}(\boldsymbol{x})$ is $x_{i}$, then $[\boldsymbol{t}(\boldsymbol{x})]_{A, e}=e_{i}$

- if $\boldsymbol{t}(\boldsymbol{x})$ is $c$ for some $c \in \mathcal{C}$, then $[\boldsymbol{t}(\boldsymbol{x})]_{A, e}=I(c)$

- if $\boldsymbol{t}(\boldsymbol{x})$ is $f(\boldsymbol{s}(\boldsymbol{x}))$ where $f \in \mathcal{R}$, and where $\boldsymbol{s}$ are terms with variables $\boldsymbol{x}$, then $[\boldsymbol{t}(\boldsymbol{x})]_{A, e}=I(f)\left([\boldsymbol{s}(\boldsymbol{x})]_{A, e}\right)$ 
For any $\Sigma$-structure $A$, any atomic formula $\phi(\boldsymbol{x})=R(\boldsymbol{t}(\boldsymbol{x}))$ where $R \in \mathcal{R}$, and where $\boldsymbol{t}$ are terms with variables $\boldsymbol{x}$, and any a list $e$ of values in the domain of $A$ at least as long as $\boldsymbol{x}$, if $[\boldsymbol{t}(\boldsymbol{x})]_{(A, e)}$ is in $I(R)$, we say that $A$ is a model of $\phi$, denoted by $A, e=\phi$. This definition is extended to any first order formula $\phi$ by induction on the structure of $\phi$. We say that a $\Sigma$-structure $A$ is a model of a theory $T$, denoted $A=T$, if and only if $\forall \phi \in T, A=\phi$.

We say that two formulas $\phi$ and $\psi$ a equisatisfiable in a given model $M$ if for any context $e,(M, e \models \phi$ if and only if $M, e=\psi)$.

We say that a theory $T$ admits semantic quantifier elimination, if for every $\phi \in \mathcal{F}(\Sigma)$, there exists $\psi \in \mathcal{F}(\Sigma)$ such that $\psi$ is quantifier free and for any model $M$ of $T$, and for any list $e$ of values, $M, e \models \phi$ iff $M, e \models \psi$. In this work, we formalize the property of semantic quantifier elimination for the theory of algebraically closed fields. We discuss further this point in section 5 .

The theory of algebraically closed fields. The signature we use in this paper to define the theory of fields (and algebraically closed fields) is $\Sigma_{\text {Fields }}=$ $\{0,1\} \cup\left\{-, .^{-1},+, *\right\} \cup \emptyset$ (so the only atoms are equalities). We will also use $\Sigma_{\text {Rings }}=\{0,1\} \cup\{-,+, *\} \cup \emptyset$. The CoQ formalization features an extra unary relation symbol for units, because the field theory is built by extending the one of rings with units. We have omitted here this extra predicate for sake of readability. We call first order theory of algebraically closed field, the set $T_{\text {ClosedFields }}$ of axioms of fields plus an axiom scheme explaining that any monic univariate polynomial with coefficients in an algebraically closed field has a root. This axiom scheme $\left(A_{n}\right)_{n \in \mathbb{N}}$ defines a countable set of axioms, one per degree of polynomial:

$$
\left(A_{n}\right):=\forall a_{0}, \ldots a_{n-1}, \exists x, x^{n}+a_{n-1} x^{n-1} \cdots+a_{1} x+a_{0}=0
$$

Theorem 1. $T_{\text {ClosedFields }}$ admits quantifier elimination.

This result is attributed to Tarski [19]. The corresponding geometrical formulation of this result, stating that projections of constructible sets are constructible sets is known as Chevalley's Constructibility theorem [7].

\section{$2.2 \quad$ Formalization issues}

In a type theoretic proof assistant like the CoQ system, it is a common practice to define the interface of an algebraic structure using record types. Here is an example of a possible definition for commutative groups:

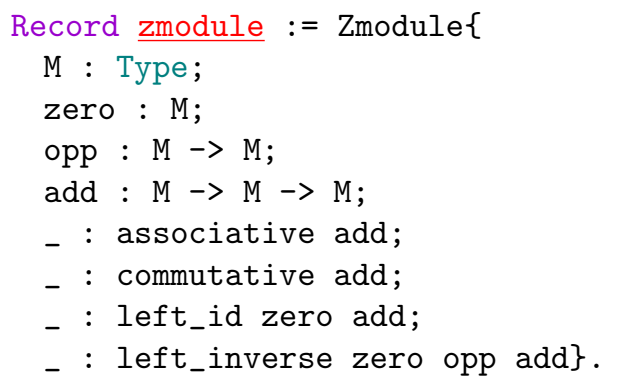


Packaging such structures can be delicate, and record nesting should be used to achieve sharing and inheritance between structures $[9,8]$. One can define similar specifications for more complex algebraic structures like ring, integralDomain and field. In our setting [8], the structure closedField of algebraically closed field is formally defined by packing a structure of field with the following extra axiom schema:

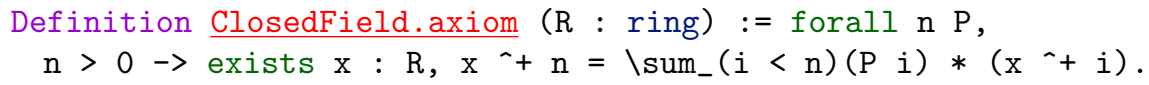

where the notation $\left(\mathrm{x}^{-}+\mathrm{n}\right)$ stands for $x^{n}$, and the right hand side of the equation is an iterated sum [5] forming the polynomial expression whose coefficients are given by the ( $P$ : nat $\rightarrow R$ ) function.

The syntactic equality predicate on such algebraic structures need not to be defined, as Coq provides such a default equality on any type. In Coq this equality is not decidable in general, and one cannot base a case analysis on two terms being equal or not. However in all what follows, we restrict our study to the case of discrete structures, in particular discrete fields. This means that we assume that there is a boolean equality test exactly reflecting CoQ equality on the terms. For instance a classical formalization of real numbers could fit this framework through the assumption of a boolean equality test, and so could a constructive formalization of algebraic numbers.

The definition of an algebraic structure interface type like zmodule can be seen as the definition of a signature $\Sigma$, together with some axioms $E$, shallow embedded in CoQ logic. In the case of zmodule, the signature is $\{0\} \cup\{-,+\} \cup \emptyset$, and the axioms are the expected ones. Populating such a type, i.e. building an element ( $Z$ : zmodule) is providing a carrier and an interpretation function, hence a structure $Z$, together with a proof that it satisfies the set of axioms

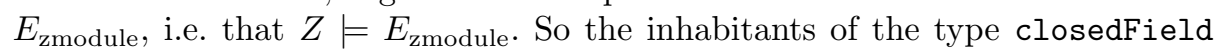
are a shallow embedding of the models of $T_{\text {ClosedFields. }}$ To state a quantifier elimination result, we also need to provide an abstract representation of first order formulas. Terms in $\mathcal{T}\left(\Sigma_{\text {Fields }}, \mathbb{N}\right)$ are formally described as the inhabitants of the following inductive type:

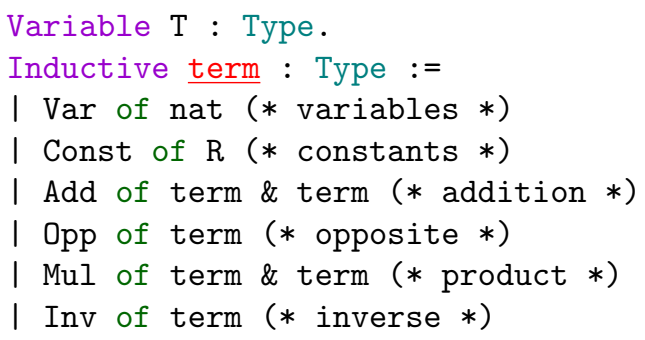

where we reflect division by the product by an inverse. Similarly, first order formulas in $\mathcal{F}\left(\Sigma_{\text {Fields }}, \mathbb{N}\right)$ are defined by:

Inductive formula : Type :=
| Bool of bool 


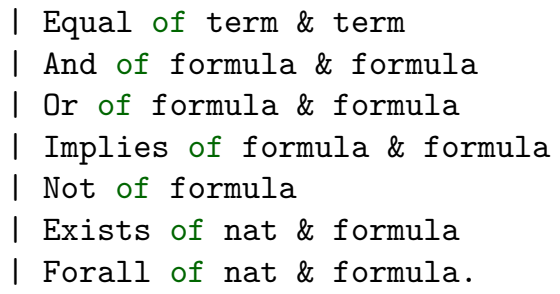

where quantifiers explicitly take as argument the name of the variable they bind. We now define a CoQ predicate holds: forall F : field, seq F $\rightarrow$ formula $\mathrm{F} \rightarrow$ Prop, such that (holds $\mathrm{F}$ e f) is $F, e=f$ (see section 2.1). This requires the definition of the eval: forall $F$ : field, seq $F \rightarrow$ term $\mathrm{F} \rightarrow \mathrm{F}$ function interpreting terms as element in the model with respect to a context, such that (eval $\mathrm{F}$ e $\mathrm{t}$ ) formalizes $[t]_{(F, e)}$. For instance, the interpretation of the abstract formula:

'forall 'X_0, 'forall 'X_1, 'forall 'X_2, 'exists 'X_3,

'X_0 *'X_3 *'X_3 + 'X_1 *'X_3 + 'X_2 == 0 : formula $\mathrm{F}$

where some notations pretty-print the constructors of the formula inductive type, is the COQ proposition:

forall a b c, exists $\mathrm{x}, \mathrm{a} * \mathrm{x} * \mathrm{x}+\mathrm{b} * \mathrm{x}+\mathrm{c}=0$ : Prop

For any $\mathrm{T}$ : Type, it is straightforward to test if a formula ( $t$ : formula $\mathrm{T}$ ) is quantifier free: we just recursively test that $t$ does not feature any Exists or Forall constructor. This results in a boolean test:

Definition qf_form : forall $\mathrm{T}$ :Type, formula $\mathrm{T} \rightarrow$ bool.

Now the CoQ theorem we prove is that there exists a transformation:

Definition q_elim : forall F : closedField, formula F $\rightarrow$ formula F such that:

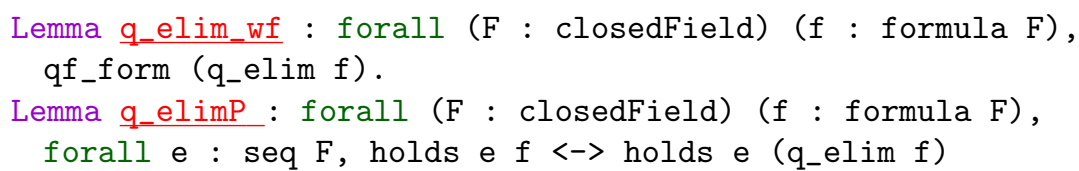

This latter theorem is a formalization of the semantic quantifier elimination, assuming that the shallow formalization of models encompasses all models of a given structure.

\subsection{Quantifier elimination by projection}

For the discrete structures we are interested in, and more generally for first order theories with decidable atoms, the elimination of a single existential quantifier entails full quantifier elimination. We give here a formal account of this reduction, for the special case of the theory of discrete rings. 
We first show that this problem is sufficiently general by encoding the theory of discrete fields into the one of discrete rings. Atoms of the abstract formulas of the discrete field theory are transformed in the following way:

- Right-hand sides are set at 0 by transforming $\left(t_{1}=t_{2}\right)$ into $\left(t_{1}-t_{2}=0\right)$

- Divisions in the left-hand sides are recursively removed by introducing quantifications over fresh variables: $C\left[t^{-1}\right]=0$ is transformed into:

$$
\begin{aligned}
& \forall x,((x * t-1=0 \wedge t * x-1=0) \vee x=t \wedge \neg(\exists x,(x * t-1=0 \wedge t * x-1=0)) \\
& \Longrightarrow(C[x]=0)
\end{aligned}
$$

This transformation to_rformula is lifted recursively to any (non atomic formula). We prove that it preserves the semantic of ring formulas in any model of the structure of ring with units ${ }^{2}$. For sake of convenience, we introduce a special data-structure for normalized quantifier-free formulas. They can be represented in disjunctive normal form as:

$$
\bigvee_{l \in L}\left(\bigwedge_{i \in I} t_{i}=0 \wedge \bigwedge_{j \in J} \neg\left(t_{j}=0\right)\right)
$$

and hence encoded by a list (or sub-formulas in the disjunction), of pairs (one for positive and one for negated atoms) of lists of terms (the left hand sides) : ( seq $(($ seq term $R) *($ seq $($ term $R)))$. We consider a field $F$, equipped with an operator:

Variable proj : nat $\rightarrow$ seq (term F) * seq (term F) $\rightarrow$ formula F.

whose first integer argument represents the name of a variable, second argument is a quantifier free conjunctive formula, and which computes a new abstract formula. This operator is meant to eliminate a quantifier from any formula of the form:

$$
\exists x_{n}, \bigwedge_{i \in I} t_{i}=0 \wedge \bigwedge_{j \in J} \neg\left(t_{j}=0\right)
$$

We hence require that on a formula on the ring signature, this operator always computes a quantifier free formula on the ring signature:

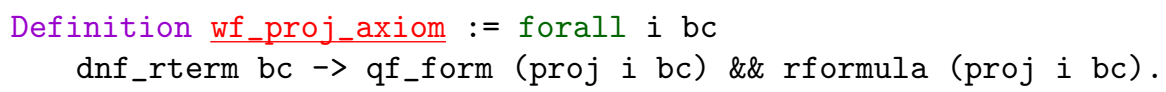

and that it computes a formula equivalent to its input:

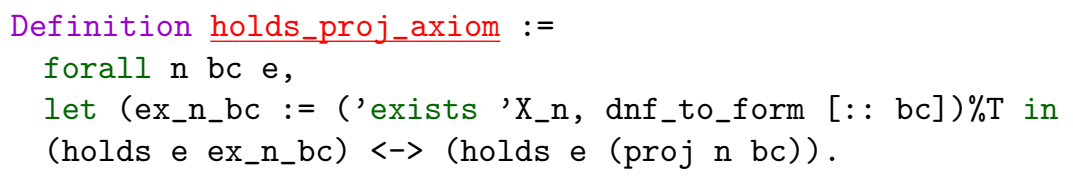

where dnf_to_form converts back the convenient representation we introduced for DNF quantifier free formulas to an inhabitant of the type formula F.

\footnotetext{
$\overline{2}$ not required to be commutative.
} 
Under the assumptions that the proj operator satisfies the properties wf_proj_axiom and holds_proj_axiom, we can now prove that the field $F$ enjoys a full quantifier elimination, meaning that we can implement the q_elim function of section 2.3. This quantifier elimination procedure proceeds by recursion on the structure of the formula, eliminating the inner-most quantifier:

- if it is an existential quantifier, the formula has the form $\exists x_{n} F$, where $F$ is quantifier-free. It converts $F$ is DNF and uses the fact that: $\exists x, \bigvee(\wedge p \wedge \wedge \neg q)$ is equivalent to $\bigvee(\exists x,(\bigwedge p \wedge \bigwedge \neg q))$ to map the proj operator on all the conjunctions of literals.

- if it is an universal quantifier, the formula has the form $\forall x_{n} F$, where $F$ is quantifier-free. It converts $\forall F$ in $\neg \exists \neg F$, and converts $\neg F$ into DNF before using again the proj operator. This transformation is valid since the decidability of atoms implies that the full theory is classical.

The sequential representation of quantifier free formulas has been introduced to ease the DNF conversions, and their combination with negations in the case of universal quantifiers.

Finally we obtain a full formal proof that if a field is equipped with a proj operator, with a proof of the two wf_proj_axiom and holds_proj_axiom properties, then we can derive a correct quantifier elimination procedure q_elim : formula $F \rightarrow$ formula $F$, which transforms any first order formula into a quantifier-free one, and such that the input and the output of the quantifier elimination are equisatisfiable in any model of a ring with units.

This generic process does not intend to provide an efficient decision procedure in general. The possibly numerous conversions in DNF make indeed this method very expensive, at least a tower of exponentials in the number of quantifiers. One can in general improve this complexity using decision procedures which are not based on quantifier elimination, and are more tractable, at least in practice. For instance, the standard way of deciding the universal theory of an algebraically closed field is to use Gröbner bases [6].

\subsection{Decidability}

The first order theory of a field is decidable if one can construct a boolean operator: $\mathrm{s}$ : seq $\mathrm{R} \rightarrow$ formula $\mathrm{R} \rightarrow$ bool, which reflects the satisfiability of any formula, i.e. satisfies the following property:

Variable F : field.

Definition DecidableField.axiom (s : seq F $\rightarrow$ formula F $\rightarrow$ bool):= forall e $f$, (holds e f) $<->$ (s e $f=$ true).

This provides a computational characterization of decidability since $s$ can be seen as a decision procedure for the first order theory of $F$.

Of course not all fields have a decidable first order theory: for instance the field theory of rational numbers is undecidable [17]. However quantifier elimination entails decidability for any first order theory with decidable atoms. It 
is hence straightforward to construct by structural recursion a boolean test qf_eval which correctly reflects the validity of such a quantifier free abstract formula (and remains unspecified on quantified formulas). The correctness of this boolean test is expressed by the lemma:

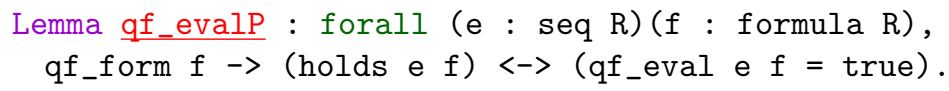

where $\mathrm{gf}_{\text {f }}$ form tests that an abstract formula does not contain any quantifier. The function

Definition proj_sat e $f:=$ qf_eval e (q_elim f).

takes a formula, eliminates its quantifiers, and applies the boolean satisfiability test qf_eval on the result. It is a correct decision procedure as shown by the formal proof that it satisfies the DecidableField.axiom specification.

\section{Polynomial arithmetic}

In section 2.3, we have shown that quantifier elimination on the first order language of fields reduces to the one over the signature of rings. This simplification aims at dealing with terms that are polynomial expressions in the variables instead of fractions. The price to pay for this reduction is that division is no longer available, even on constant coefficients, even if in fact they are interpreted in a field. The semantic results we can use should hence be provable in polynomial rings of the form $R[X]$, where $R$ is only an integral domain. In this section, we give a formal account of the results on polynomial arithmetic needed in the sequel.

\subsection{Representation}

We represent univariate polynomials as lists of coefficients with lowest degree coefficients in head position. We require polynomials to be in normal form, in the sense that the last element of the list is never zero. Hence the type \{poly T \} of polynomials with coefficients in the type $\mathrm{T}$ is a so-called sigma type, which packages a list, and a proof that it last element is non zero. The zero polynomial is therefore represented by the empty list, with a default zero value for the head constant function.

It is convenient and standard to define the degree of a univariate monomial as its exponent, except for the zero constant, whose degree is set at $-\infty$. Then the degree of a polynomial is the maximum of the degree of its monomial. To avoid introducing option types, we simply work here with the size of a polynomial, which is the size of its list. This lifts the usual codomain of degree from $\{-\infty\} \cup \mathbb{N}$ to $\mathbb{N}$ since in our case:

$$
\operatorname{size}(p)= \begin{cases}0 & , \text { if and only if } p=0 \\ \operatorname{deg}(p)+1 & , \text { otherwise }\end{cases}
$$




\subsection{Pseudo-divisions, pseudo-gcd}

As soon as coefficients are equipped with a structure of field, one can program the well-known algorithm of Euclidean division, and define and specify greater common divisor. Here we have a weaker assumption on the coefficients: we only benefit from ring operations, but we still know that this ring is an integral domain. If integral domains are a natural setting for the study of divisibility, Euclidean division is no more possible in general. Indeed this division algorithm involves division among the coefficients of the respective polynomials, which could not be possible inside the integral domain. However it might still remain doable if a sufficient power of the leading coefficient of the divisor divides all the coefficients of the dividend. For instance one cannot perform Euclidean division of $2 X^{2}+3$ by $2 X+1$ in $\mathbb{Z}[X]$, but one can divides $2 X^{2}+6$ by $2 X+1$ in $\mathbb{Z}[X]$. In the context of integral domains, Euclidean division should be replaced by pseudo-division.

Definition 1 (Pseudo-division). Let $R$ be an integral domain. Let $P=a_{p} X^{p}+$ $\ldots a_{0} \in R[X]$ and $Q=b_{q} X^{q}+\ldots b_{0} \in R[X]$. We define the pseudo-division of $P$ by $Q$ as the Euclidean division of $b_{q}^{d} P$ by $Q$, where $d$ is the smallest integer such that the division is possible inside $R[X]$.

Note that $d$ always exists and is at most $p-q+1$. We denote it (scalp $p q$ ). The Euclidean pseudo-division also returns the pseudo-quotient divp, denoted $\% /$, and the peusdo-remainder modp, denoted $\% \%$, satisfying the following specification:

Lemma divp_spec : forall p q,

$(\operatorname{scalp} \mathrm{p} \mathrm{q}) \%: P * \mathrm{p}=\mathrm{p} \% / \mathrm{q} * \mathrm{q}+\mathrm{p} \% \mathrm{q}$.

Note that in general, the correcting coefficient scalp p q could be smaller than a power of the leading coefficient. The smallest value is in fact a power of the content of the divisor. All the possible choices in the correcting factor lead to different (but associated) values in the pseudo-quotient and remainder.

We say that $\mathrm{p}$ pseudo-divides $\mathrm{q}$, denoted ( $\mathrm{p} \% \mathrm{l} \mathrm{q}$ ) if the pseudo-remainder of $\mathrm{p}$ by $\mathrm{q}$ is zero. We recover some standard lemmas about divisibility like:

Lemma dvdp_mul : forall d1 d2 m1 m2 : \{poly R\}, $\mathrm{d} 1 \%|\mathrm{~m} 1 \rightarrow \mathrm{d} 2 \%| \mathrm{m} 2 \rightarrow \mathrm{d} 1 * \mathrm{~d} 2 \% \mid \mathrm{m} 1 * \mathrm{~m} 2$.

The pseudo greatest common divisor gcdp is obtained by replacing division by pseudo-division in the Euclidean algorithm. This is not the optimal algorithm to compute such a greatest common divisor, which is a non trivial problem. We choose here a naive implementation, since at this point, we are not concerned with efficiency. However we recover standard properties of the standard greatest common divisor, like:

Lemma root_gcd : forall p q x,

$\operatorname{root}(\operatorname{gcdp} p q) \times=\operatorname{root} p \times \& \& \operatorname{root} q x$.

where root $\mathrm{p} x$ means that $\mathrm{p}$ evaluates to 0 at the value $\mathrm{x}$. 


\subsection{Common roots, exclusive roots}

Recall from section 2.3 that we aim at eliminating the existential quantifier from a formula of the form:

$$
\text { (1) } \exists x \in R, \bigwedge_{i=1}^{n} P_{i}(x)=0 \wedge \bigwedge_{j=1}^{m} Q_{j}(x) \neq 0
$$

Indeed, after the reduction from the first order theory of fields to the first order theory of ring, and the normalization of atoms, atoms are zero conditions on polynomial expressions. In other words, given two finite families of polynomials $\left(P_{i}\right)$ and $\left(Q_{j}\right)$, we need to decide if there exists a point in the underlying field which is a common root of the $\left(P_{i}\right)$ s but root of no $Q_{j}$ :

$$
(1) \Leftrightarrow \exists x \in R,\left(\operatorname{gcd}_{i=1}^{n} P_{i}\right)(x)=0 \wedge\left(\prod_{i=1}^{m} Q_{i}\right)(x) \neq 0
$$

Given two polynomials $P$ and $Q$ with coefficients in an integral domain $R$, we introduce the greatest divisor of $P$ coprime to $Q$, denoted $\operatorname{gdco}_{Q}(P)$ and recursively defined as:

$$
\operatorname{gdco}_{Q}(P)= \begin{cases}1 & , \text { if } P=0 \wedge Q=0 \\ 0 & , \text { if } P=0 \wedge Q \neq 0 \\ P & , \text { if } \operatorname{gcdp} P Q=1 \\ \operatorname{gdco}_{Q}(P / \operatorname{gcdp} P Q) & , \text { otherwise }\end{cases}
$$

where the quotient is in fact a pseudo-quotient. In particular, $\operatorname{gdco}_{Q}(P)$ satisfies the following property:

$$
\exists x \in R, P(x)=0 \wedge Q(x) \neq 0 \Leftrightarrow \exists x \in R, \operatorname{gdco}_{Q}(P)(x)=0
$$

Introducing this $\operatorname{gdco}_{Q}(P)$ operator provides a new equivalent to (1):

$$
(1) \Leftrightarrow \exists x \in R, \operatorname{gdco}_{\left(\prod_{i=1}^{m} Q_{i}\right)}\left(\operatorname{gcd}_{i=1}^{n} P_{i}\right)(x)=0
$$

which in particular simplifies the one used in [2]. But if the underlying field is algebraically closed, then any non zero polynomial has a root as soon as it is non constant, hence:

$$
(1) \Leftrightarrow \operatorname{size}\left(\operatorname{gdco}_{\left(\prod_{i=1}^{m} Q_{i}\right)}\left(\operatorname{gcd}_{i=1}^{n} P_{i}\right)\right) \neq 1
$$

But this is not a first order formula. Indeed, size, gcd and gdco are not directly expressible as terms in $\mathcal{T}\left(\Sigma_{\text {Ring }}, \mathbb{N}\right)$ because they may depend on other variables than $x$. For example, consider $\phi(y):=\exists x, x=0 \wedge x y \neq 0$. We know that:

$$
\phi(y) \Leftrightarrow \operatorname{size}\left(\operatorname{gdco}_{x y}(x)\right) \neq 1
$$




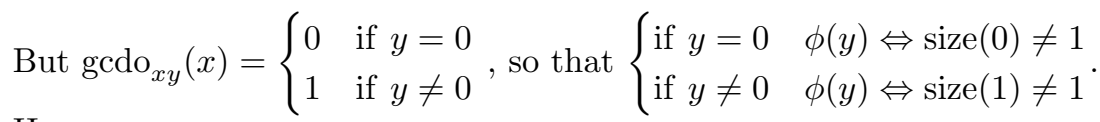

Hence:

$$
\phi(y) \Leftrightarrow((\operatorname{size}(0) \neq 1) \wedge(y=0)) \vee((\operatorname{size}(1) \neq 1) \wedge(y \neq 0))
$$

The same transformations apply to size so that in the end:

$$
\phi(y) \Leftrightarrow((\neg \text { false }) \wedge(y=0)) \vee((1 \neq 1) \wedge(y \neq 0))
$$

In the general case, the translation of (2) into a first order formula uses case analysis, in fact zero tests, on the coefficients of the polynomials $P_{i}$ and $Q_{i}$. The final first order formula is a disjunction compounding the first order characterizations obtained for each case. In the next part we present the algorithm that systematizes this case analysis and reconstruction of formulas.

\section{Quantifier elimination for algebraically closed fields}

Let $\mathcal{P}, \mathcal{Q} \subset \mathcal{T}\left(\Sigma_{\text {Rings }},\left\{x_{1}, \ldots x_{n}\right\}\right)$ two finite sets of terms. In this section, we describe how to effectively transform a formula:

$$
\phi:=\exists x_{k}, \bigwedge_{p \in \mathcal{P}} p=0 \wedge \bigwedge_{q \in \mathcal{Q}} \neg(q=0)
$$

over $\Sigma_{\text {Rings }}$ into a quantifier free formula $\psi$ in $\mathcal{F}\left(\Sigma_{\text {Rings }},\left\{x_{1}, \ldots x_{n}\right\}\right)$ such that:

$$
\forall M \models T_{\text {ClosedField }}, \forall e \in M^{n}, \quad(M, e) \models \phi \Leftrightarrow(M, e) \models \psi
$$

Section 3.3 describes how different values for the context lead to different candidates for the quantifier free formula $\psi$. It is still possible to construct such a $\psi$ because we can construct an algebraic, quantifier free, model-independent description of a finite partition of the space of parameters into cells. Each cell corresponds to a uniform characterization $\psi$ in the language of rings. The description of this partition is obtained by analyzing the tree of successive zero tests performed when computing the degrees and the pseudo divisions involved in the expression (2) of section 3.3.

A term $t \in \mathcal{P} \cup \mathcal{Q}$ can be seen as polynomials in $R\left[x_{1}, \ldots, x_{n}\right]$. Up to ring theory, we can even reorder the sub-terms of $t$, to factorize the powers of $x_{k}$, At this syntactic level, we introduce the type of formal polynomials, defined as lists of ring terms:

Definition polyF $(\mathrm{T}:$ Type) := seq (term $\mathrm{T}$ ).

We also define the function:

Definition abstrX ( $R$ : Ring) (i : nat) ( $t$ : term $R$ ) : (polyF $R$ ) := $\cdots$ 
by induction on a ( $t$ : term $F$ ), which computes the formal polynomial associated to $t$ seen as a polynomial in the variable $x_{i}$. A formal polynomial $t$ can be interpreted as a univariate polynomial $\left[p_{t}\right]_{e}$ given a large enough context:

Fixpoint eval_poly ( $R$ : Ring) (e : seq R) (pf : polyF R) := if pf is c : : qf then (eval_poly e qf)*'X + (eval e c) \%:P else 0 .

We need to define again all the operations we have used in the informal presentation of section 3 like the size and greatest common division, to make them operate of formal polynomials. We moreover expect this transformation to be semantically sound. For instance, for a function ( $f$ : poly $\mathrm{R} \rightarrow \mathrm{A}$ ), with an arbitrary return type $\mathrm{A}$, we could ask its formal counterpart $\left(F_{f}\right.$ : term $\mathrm{F} \rightarrow \mathrm{A}$ ) to satisfy:

$$
\forall t \in \mathcal{T}\left(\Sigma,\left\{x_{1}, \ldots x_{n}\right\}\right), \forall M \models T_{\text {ClosedFields }}, \forall e \in M^{n}, f\left(\left[p_{t}\right]_{e}\right)=\left[F_{f}(t)\right]_{e}
$$

This is unfortunately not possible: consider the size function on polynomials, applied to the polynomial $x$. According to the value assigned by a given context to $x$, the size of $x$ will be either 0 or 1 . But there is no way to encode a case analysis at the syntactic level of terms handled by a formal counterpart $F_{\text {size }}$. In fact, these formal functions on terms should return lists of values, reflecting all the possible tests the body of the polynomial function performs. Going back to section 3.3, the output quantifier free formula is a disjunction over all the different values of the degree, specified by the conditions leading to these respective values. This construction requires inspecting the invariants of the code of the functions, to prove the correctness and soundness of the generated conditions. This approach is the one usually described in the literature (see e.g. [2]).

To avoid this painful formula reconstruction, we diverge from this standard presentation. First, we transform polynomial operations to return formulas instead of terms. This allows to encode case analysis, using the simple construction:

Definition ifF (then else cond: formula F) : formula $F$ :=

((cond 八 then) $\backslash((\sim$ cond $) 八$ else $)) \%$ T.

Simultaneously, we concisely internalize the administration of conditions in the body of the formal counterpart by programming them in continuation passing style (CPS). The CPS version of a function $f: A_{1}->\ldots \rightarrow A_{n}->$ B has the form $f_{-} \operatorname{cps}(\mathrm{k}: \mathrm{B} \rightarrow \mathrm{T})\left(\mathrm{a}_{1}: \mathrm{A}_{1}\right) \ldots\left(\mathrm{a}_{n}: \mathrm{A}_{n}\right): \mathrm{T}$, where $\mathrm{k}$ is called the continuation. For example, the rewritten size function is

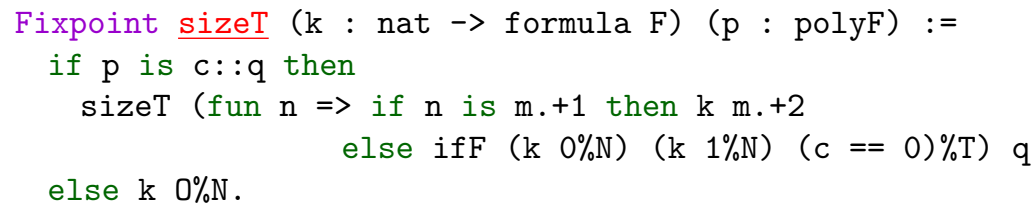

which is a straightforward translation of a CPS version of the size function over the polynomials of section 3.1. Note that working with continuations means that 
the code handles the formulas to be output, instead of the arguments, and can hence feature the iff construction to directly build the case disjunction in the language of formulas. The zero test on formal polynomial is then:

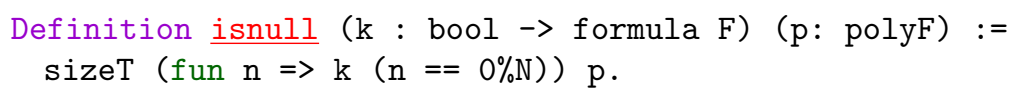

The semantic specification that a CPS function

fT : (B $\rightarrow$ formula $F) \rightarrow\left(A_{1}->\ldots \rightarrow A_{n}->\right.$ formula $\left.F\right)$ which builds the formal counterpart of a function $f: A_{1}->\ldots \rightarrow A_{n}->B$ is formally expressed by a lemma of the form:

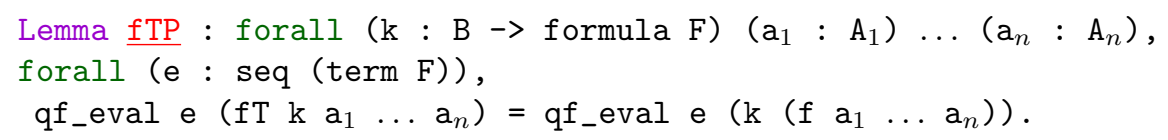

For example, sizeT is correct w.r.t. size since we prove that:

Lemma sizeTP : forall k p e, qf_eval e (sizeT k p) = qf_eval e (k (size (eval_poly e p))).

We transform and specify the gdcop operation of 3.3 in the same CPS way to obtain a gcdpt function, naturally extended to lists of formal polynomials by gdcopTs. And we can express a first quantifier elimination lemma, that takes a list of formal polynomials ps and a formal polynomial q:

Definition ex_elim_seq (ps : seq polyF) (q : polyF) : formula F := gcdpTs (gdcopT q (sizeT $($ fun $n \Rightarrow$ Bool $(n !=1 \% N)))$ ) ps.

where Bool is a constructor of formula F.

The code can be read using monadic operations (this is no more CoQ code) as :

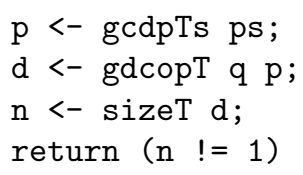

The projection function required at section 2.3 in finally implemented as:

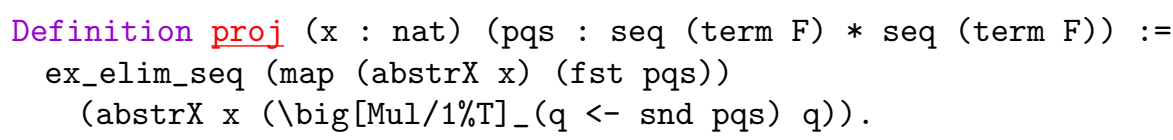

where \big is a notation for iterated operators [5], which constructs the formal term representing the product of the polynomials coming from the list (snd pqs ). Proving that the proj operator outputs quantifier free formulas is straightforward: we have to check that each continuation can only output quantifier free formula. This is done by a trivial case analysis on each of the continuations. Proving its semantic correctness, i.e. the holds_proj_axiom axiom of section 2.3 , is a combination of the semantic correctness of the CPS functions with the results formally proved in section 3.3 . 


\section{Conclusion}

From a model-theoretic point of view, our approach deserves further discussion. In this work, we weaken the syntactic characterization of quantifier elimination given in section 2.1 along two directions. The first one is that our shallow approach only capture a semantic characterization of the equivalence between formulas: working at the abstract level of equivalence within the first order language of fields would require a deep embedding of the first order provability predicate. In the current formalization, nothing prevent us to use higher-order features in our proofs of semantic equivalence. An other option would be to provide a formal proof of Gödel's completeness theorem [12]. This result has already been formalized within the Isabelle system [16]. It would be interesting to investigate the work needed to come up with a similar result in CoQ. We could for instance rely on the structures already introduced for a CoQ formal proof of Gödel's incompleteness theorem [14], and the constructive approaches to the Gödel's completeness result [3]. Yet this would also require us to deeper formalize the $=$ relation, and to prove formally that our shallow approach captures all the models of a given structure. Again there are here at least two options. The first one is to consider only models that can be defined in CoQ, which should lead to a straightforward proof. A more general option would be to consider all the models definable in set theory, possibly relying on previous works about the formalization of set theory in CoQ $[1,18]$.

However, one of our main motivation was to provide a convenient framework for the user to get a proof-producing decision procedure for the first-order theory of algebraically closed fields. We have not proved correct an efficient procedure, but this was not our purpose. Efficient decision procedures for the theory of real closed fields usually best deal with the universal fragment of the theory using Gröbner bases computations. Harrison [10] has formalized both quantifier elimination for the theory of complex numbers, and a proof producing version of Buchberger algorithm [6]. An efficient Gröbner based tactic is also available in CoQ [15] for complex numbers.

The reduction of quantifier elimination to the elimination of a single existential is a standard result. Nipkow proposes in [13] a modular framework to build decision procedures along this motive. However, the continuation passing style we use to feed the prerequisite existential elimination seems an original idea. This approach makes the programing and the specification elegant and concise. Moreover, the produced formulas are in general more compact than the one produced by an invariant-based approach.

To the best of our knowledge, the present work is the first to address formally quantifier elimination for a generic structure of algebraically closed fields. We plan to investigate how the method we have presented scales to the theory of real closed fields, which is very similar in their structure [2].

Acknowledgments The authors wish to thank Georges Gonthier for numerous comments and improvements. The proofs relating quantifier elimination and 
decidability were done in close collaboration with him, and he suggested the successful idea of using CPS-style to transform algorithms on polynomials into formula producing ones. The authors are also grateful to Thierry Coquand for his comments and suggestions on preliminary versions of this work.

\section{References}

1. Bruno Barras. Sets in coq, coq in sets. In Proceedings of the 1st Coq Workshop. Technical University of Munich Research Report, 2009.

2. Saugata Basu, Richard Pollack, and Marie-Françoise Roy. Algorithms in Real Algebraic Geometry (Algorithms and Computation in Mathematics). Springer-Verlag New York, Inc., Secaucus, NJ, USA, 2006.

3. Stefano Berardi and Silvio Valentini. Krivine's intuitionistic proof of classical completeness (for countable languages). Ann. Pure Appl. Logic, 129(1-3):93-106, 2004.

4. Yves Bertot and Pierre Castéran. Interactive Theorem Proving and Program Development, Coq'Art: the Calculus of Inductive Constructions. Springer-Verlag, 2004.

5. Yves Bertot, Georges Gonthier, Sidi Ould Biha, and Ioana Pasca. Canonical big operators. In Theorem Proving in Higher-Order Logics, volume 5170 of LNCS, pages $86-101,2008$.

6. B. Buchberger. Groebner Bases: Applications. In A. V. Mikhalev and G. F. Pilz, editors, The Concise Handbook of Algebra, pages 265-268. Kluwer Academic Publishers, Dordrecht, Netherlands., 2002.

7. Claude Chevalley and Henri Cartan. Schémas normaux; morphismes; ensembles constructibles. In Séminaire Henri Cartan, volume 8, pages 1-10. Numdam, 19551956. http://www. numdam.org/item?id=SHC_1955-1956__8__A7_0.

8. Georges Gonthier and François Garillot and Assia Mahboubi and Laurence Rideau. Packaging mathematical structures. In Stefan Berghofer, Tobias Nipkow, Christian Urban, and Makarius Wenzel, editors, TPHOLs, volume 5674 of Lecture Notes in Computer Science, pages 327-342. Springer, 2009.

9. Herman Geuvers, Randy Pollack, Freek Wiedijk, and Jan Zwanenburg. A constructive algebraic hierarchy in Coq. Journal of Symbolic Computation, 34(4):271-286, 2002.

10. John Harrison. Complex quantifier elimination in HOL. In Richard J. Boulton and Paul B. Jackson, editors, TPHOLs 2001: Supplemental Proceedings, pages 159-174. Division of Informatics, University of Edinburgh, 2001. Published as Informatics Report Series EDI-INF-RR-0046. Available on the Web at http://www.informatics.ed.ac.uk/publications/report/0046.html.

11. Wilfried Hodges. A shorter model theory. Cambridge University Press, 1997.

12. Kurt Gödel. Über die Vollständigkeit des Logikkalküls. $\mathrm{PhD}$ thesis, University of Vienna, Austria, 1929.

13. Tobias Nipkow. Linear quantifier elimination. In A. Armando, P. Baumgartner, and G. Dowek, editors, Automated Reasoning (IJCAR 2008), volume 5195 of LNCS, pages 18-33. Springer, 2008.

14. Russel O'Connor. Incompleteness 85 Completeness, Formalizing Logic and Analysis in Type Theory. PhD thesis, Radboud University Nijmegen, Netherlands, 2009.

15. Loic Pottier. Connecting gröbner bases programs with coq to do proofs in algebra, geometry and arithmetics. In LPAR Workshops, volume 418 of CEUR Workshop Proceedings. CEUR-WS.org, 2008. 
16. Tom Ridge and James Margetson. A mechanically verified, sound and complete theorem prover for first order logic. In Joe Hurd and Thomas F. Melham, editors, TPHOLs, volume 3603 of Lecture Notes in Computer Science, pages 294-309, 2005.

17. Julia Robinson. Definability and decision problems in arithmetic. Journal of Symbolic Logic, 14:98-114, 1949.

18. Carlos T. Simpson. Formalized proof, computation, and the construction problem in algebraic geometry, 2004.

19. Alfred Tarski. A decision method for elementary algebra and geometry. Manuscript. Santa Monica, CA: RAND Corp., 1948. Republished as A Decision Method for Elementary Algebra and Geometry, 2nd ed. Berkeley, CA: University of California Press, 1951. 